\title{
États-Unis : Qu'apprend-on dans les écoles américaines depuis 2002 ?
}

Le point sur...

Bénédicte Robert

\section{CpenEdition}

\section{Journals}

Édition électronique

URL : https://journals.openedition.org/ries/1118

DOI : 10.4000/ries. 1118

ISSN : 2261-4265

Éditeur

France Education international

Édition imprimée

Date de publication : 1 septembre 2006

Pagination : 10-13

ISSN : $1254-4590$

Référence électronique

Bénédicte Robert, «États-Unis : Qu'apprend-on dans les écoles américaines depuis 2002 ? », Revue internationale d'éducation de Sèvres [En ligne], 42 I septembre 2006, mis en ligne le 15 novembre 2011, consulté le 06 juillet 2021. URL : http://journals.openedition.org/ries/1118 ; DOI : https://doi.org/ 10.4000/ries. 1118

Ce document a été généré automatiquement le 6 juillet 2021.

(c) Tous droits réservés 


\title{
États-Unis : Qu'apprend-on dans les écoles américaines depuis 2002 ?
}

Le point sur...

\author{
Bénédicte Robert
}

1 En mars de cette année, le New York Times consacrait un long article à l'une des conséquences de la loi fédérale No Child Left Behind (NCLB) signée par George W. Bush en $2002^{1}$. En s'appuyant sur les conclusions d'un rapport du Center on Education Policy (CEP), centre de recherche indépendant, le journaliste argumentait que la loi obligeait les établissements scolaires américains à se concentrer presque exclusivement à l'enseignement de l'anglais et des mathématiques, sacrifiant donc notamment les sciences sociales, les disciplines artistiques et les langues étrangères ${ }^{2}$. L'anglais et les mathématiques font en effet l'objet d'examens de fin d'année avec de grands enjeux (high-stakes testing), à la fois pour les élèves qui ne peuvent recevoir leur diplôme de fin de cycle sans les avoir réussis, pour les principaux dont le contrat à la performance en dépend, et même pour les enseignants qui, dans certains cas, peuvent être licenciés. Un tel système de sanctions incite donc la majorité des acteurs du système éducatif à réussir dans ces deux disciplines, et pour cela, à y consacrer l'essentiel de leur temps.

2 Cette situation est tout à fait nouvelle. En effet, le programme scolaire américain est historiquement moins dense que large ; il n'était par exemple pas si rare de trouver des cours de menuiserie ou de couture dans les lycées américains d'enseignement général il y a seulement quelques années. L'acquisition de connaissances n'est pas l'objectif unique de la common school américaine - équivalent historique de l'école républicaine qui vise aussi à faire des élèves des citoyens responsables et autonomes ${ }^{3}$. Pendant tout le $\mathrm{XX}^{\mathrm{e}}$ siècle, le système américain a notamment enseigné les valeurs d'initiative, d'ingéniosité, et d'intégrité, l'esprit d'équipe et le sens de l'effort, valeurs qui selon certains analystes ont aujourd'hui malheureusement disparu des établissements scolaires. Comment en est-on arrivé là ?

3 L'historienne américaine Patricia Graham montre qu'après avoir eu pour objectif l'assimilation (1900-1920), l'éveil de l'enfant (1920-1954) puis l'accès de tous les élèves à l'éducation (1954-1983), la mission de l'école américaine pour la scolarité obligatoire 
est, depuis 1983, la réussite scolaire (achievement), mesurée par des examens standardisés ${ }^{4}$. En effet, en 1983, le rapport A nation at risk peignait un tableau très noir des connaissances des élèves américains et faisait naître le sentiment d'une crise de l'éducation conduisant à une crise de l'économie américaine ${ }^{5}$. La réforme dite des standards, qui transforme progressivement la vie des établissements américains depuis vingt ans, propose de résoudre cette crise par un recentrage de l'éducation sur les contenus, définis comme des compétences plus que comme des connaissances. En effet, la réforme a été poussée moins par les acteurs du champ éducatif que par les gouverneurs des États, soucieux de répondre aux attentes de leur électorat et surtout à celles des entreprises en manque de main-d'œuvre qualifiée leur permettant d'être compétitives ${ }^{6}$. Ces compétences sont évaluées par des examens, et des sanctions s'appliquent (principe d'accountability) en cas de résultats insuffisants.

Ce triptyque modifie considérablement l'enseignement aux États-Unis, mais de façon variable suivant les États puisqu'à la suite d'un grand débat national, les réformateurs ont échoué à imposer cette réforme au plan national, ce qui aurait unifié les compétences exigibles ainsi que les examens ${ }^{7}$. Des États aussi différents politiquement que le Texas, le Colorado, la Floride et le Massachusetts ont constitué depuis le début des années 1990 l'avant-garde de cette réforme, tandis que d'autres, comme le Maine, le Vermont et le New Hampshire, ne s'y sont presque pas impliqués. Dans certains cas, les districts sont allés plus loin que les États ; c'est le cas par exemple de Chicago (Illinois) depuis 1996. NCLB oblige désormais tous les États à adopter la réforme des standards, mais elle ne les oblige pas à adopter les mêmes exigences.

5 Deux questions se posent : en quoi la réforme des standards, par son insistance sur les compétences et les examens, a-t-elle pu se traduire, selon certains auteurs, par un rétrécissement de l'éducation? Plus spécifiquement, dans quelle mesure la loi NCLB a-telle réduit l'éducation à l'enseignement de l'anglais et des mathématiques?

6 La réponse à la première question ne nécessite pas d'examiner la loi fédérale. En effet, celle-ci a été mise en œuvre pour la première fois en 2002-2003, tandis que certains districts ont une pratique plus ancienne de la réforme des standards et de l'accountability. Le district de Chicago (435 000 élèves) a fait l'objet de nombreuses études puisque la réforme y a été effective pour la première fois en 1996-1997. Pauline Lipman montre ainsi que la réforme adoptée à Chicago a eu plusieurs effets négatifs ${ }^{8}$. Pour être sûrs de ne rien oublier dans le programme, les enseignants collent au plus près des manuels, parfois même l'administration leur donne un plan détaillé de chaque leçon (scripted curriculum), limitant toute liberté pédagogique. Cette pratique fait fuir les meilleurs enseignants, ou du moins les démoralise. Elle n'incite pas non plus les jeunes à entrer dans la profession. L'étiquetage de la majorité des établissements de Chicago comme établissements en échec stigmatise les établissements et nuit au moral des équipes pédagogiques. Enfin et surtout, l'examen de fin d'année induit des pratiques de bachotage (teach to the test) prenant différentes formes: pratique répétitive de questions types (drill-and-kill), entraînement au remplissage des feuilles de réponse, rétrécissement des leçons sur des compétences susceptibles d'être testées. Lipman conclut que ce genre d'éducation prépare en fait à des métiers à faible qualification. L'analyse d'Anthony Bryk insiste plutôt sur la manipulation par l'administration du district des résultats aux examens'. Pour Bryk, comme pour d'autres analystes, la réforme a permis d'aplanir la situation catastrophique du district (en matière de résultats scolaires, mais aussi de corruption 
et de violence), mais le district doit désormais insister sur la qualité de l'enseignement, à travers notamment la mise en cohérence des programmes avec les standards étatiques et le développement de la formation continue pour les enseignants ${ }^{10}$. Depuis 2002 , le district a orienté ses efforts dans cette direction.

7 Le rétrécissement de l'enseignement à l'anglais et aux mathématiques est plus directement attribuable à NCLB. La loi impose entre autres que les districts organisent des examens annuels du CE2 à la $4^{\mathrm{e}}$ en anglais et mathématiques, et au moins une fois au lycée dans ces deux disciplines. À partir de 2007, les districts devront aussi proposer un test en science à l'école primaire, au collège et au lycée. Ce nouveau rythme explique par exemple que, dans le Massachusetts qui propose des examens de rattrapage, les écoles primaires et les collèges soient mobilisés environ 37 jours par an pour l'organisation des examens. Les examens étant la base d'un système de classement (et de sanction) en vigueur sur tout le territoire (mais avec des critères propres aux États), la fin de l'année est souvent difficile. Le rapport du CEP, centre de recherche indépendant, indique que l'une des conséquences de cela est que $71 \%$ des districts ont réduit le temps d'instruction dans une discipline au moins, autre que l'anglais ou les mathématiques. L'enquête du CEP montre une différence importante dans l'enseignement de la lecture, les districts urbains y consacrant presque deux heures quotidiennement, contre une heure et demie en moyenne. Cet enseignement se fait par bloc, de préférence pendant les premières heures de la journée, et est précisément minuté, particulièrement dans les districts urbains les plus défavorisés, ceci afin de s'assurer d'un temps minimum d'instruction dans les matières à examen.

8 Enfin, pour répondre aux exigences de la loi, et toujours dans le but d'améliorer la performance des élèves aux examens, les districts et les établissements ont aussi mis en place d'autres stratégies. Les personnels administratif et pédagogique des districts travaillent à apporter plus de cohérence à l'instruction dans les classes, les enseignants se référant aux standards et aux examens étatiques. Autre point positif souligné par le rapport, les principaux savent beaucoup mieux interpréter les données produites à l'occasion des examens pour adapter l'enseignement aux besoins des élèves. L'utilisation de ces données pour guider l'instruction en est encore à ses débuts mais dans certains établissements elle permet vraiment de s'assurer que tous les élèves, et surtout les plus en difficulté, bénéficient d'un enseignement de qualité.

9 La loi doit être révisée par le Congrès en 2007. Elle ne sera probablement pas démantelée comme certains de ses détracteurs le souhaitaient, mais plusieurs problèmes devraient être abordés, comme le temps excessif passé à préparer ou à passer les examens, la variabilité des critères de réussite entre les États, et la catégorisation démesurée des établissements en échec. 


\section{NOTES}

1. S. Dillon, «Schools cut back subjects to push reading and math », New York Times, 26 mars 2006. Voir aussi L. Emile-Besse, "Le système éducatif américain », Revue internationale d'éducation de Sèvres (35), 2004.

2. Le rapport est accessible en ligne à http://www.cep-dc.org/nclb/Year4/Press

3. Voir par exemple l'ouvrage de l'historien américain Lawrence Cremin : L.A. Cremin. The American common school, an historic conception. Teachers College Columbia University, New York, 1951.

4. P.A. Graham. Schooling America: how the public schools meet the nation's changing needs. Oxford; New York: Oxford University Press, 2005.

5. National Commission on Excellence in Education. A nation at risk: the imperative for educational reform: a report to the Nation and the Secretary of Education. Washington, D.C.: Government printing office, 1983.

6. Voir sur ce point J. Hochschild, "Rethinking accountability politics ", in P.E. Peterson \& M.R. West. No child left behind? The politics and practice of school accountability. Washington, D.C.: Brookings Institution Press, 2003, p. 107-123.

7. Voir D. Ravitch. Debating the future of American education: do we need national standards and assessments? Washington, D.C.: Brookings Institution, 1995 et J.F. Jennings. Why national standards and tests?: politics and the quest for better schools. Thousand Oaks, CA : Sage Publications, 1998 pour une histoire de ce débat.

8. P. Lipman. High stakes education: inequality, globalization, and urban school reform. New York: RoutledgeFalmer, 2004.

9. A.S. Bryk. No Child Left Behind, Chicago-style. In P. E. Peterson \& M. R. West (Eds.), No child left behind? The politics and practice of school accountability. Washington, D.C.: Brookings Institution Press, 2003, pp. 242-268.

10. A. Russo. School reform in Chicago: lessons in policy and practice. Cambridge, MA: Harvard Education Press, 2004. B. Jacob. High stakes in Chicago; Did Chicago rising test scores reflect genuine academic improvement? Education Next, 3 (1), 2003, pp. 66-72. K.K. Wong. The Big Stick. Education Next, 3 (1), 2003, pp. 44-49.

\section{INDEX}

Index géographique : États-Unis

Mots-clés : langue anglaise, mathématiques, matière d'enseignement, matière obligatoire, No Child Left Behind Act, réforme de l'enseignement, système éducatif 